experts to rationally debate the cost-effectiveness of medications rather than leave it, and all other aspects of drug availability, to the whims of the market. On June 20, 2018, Canada launched the Advisory Council on the Implementation of National Pharmacare. A national formulary could provide Dr. Eric Hoskins, chair of the advisory council, with the constitutional means to succeed, just as Justice Hall provided a pathway to medicare half a century ago.

Vivian C. McAlister, MB

Coeditor, Canadian fournal of Surgery
Competing interests: None declared. The views expressed are those of the author and do not necessarily reflect the views of employers or institutions with which the author is affiliated.

DOI: $10.1503 /$ cjs.010418

\section{References}

1. Harvey E. Trudeau government meddling in provincial mandates. Can F Surg 2017;60:4.

2. Government of Canada. Canada's Health Care System. www.canada. $\mathrm{ca} / \mathrm{en} / \mathrm{h}$ ealth-canada/services/health-care-system/reports-publications/ health-care-system/canada.html (accessed 23 June 2018).

3. Justice Emmett Hall. Farm Forum (interview), 2 Nov. 1964. CBC Digital Archives. www.cbc.ca/player/play/1801207811 (accessed 23 June 2018)

\title{
Les régimes universels d'assurance médicaments provinciaux doivent pouvoir s'appuyer sur un approvisionnement sûr et abordable : Question de sécurité nationale?
}

Les opinions exprimées dans cet éditorial sont celles de l'auteur et ne représentent pas nécessairement celles de l'Association médicale canadienne ou ses filiales.

ans les années 1930, le gouvernement ontarien a eu recours aux tribunaux pour forcer le retrait d'un dépliant du gouvernement fédéral qui mettait les parents en garde contre la surocclusion dentaire causée par la succion du pouce. Ce précédent et d'autres du même type sont suffisants pour empêcher le gouvernement fédéral de s'ingérer dans les soins de santé qui relèvent des compétences provinciales dans un système qui permet aux régions d'établir leurs propres priorités ${ }^{1}$.

La Commission Hall était au courant de cette restriction lorsqu'elle a ouvert la voie à un régime public de soins de santé, officialisé par l'adoption de la Loi sur les soins médicaux de 1966 qui s'ajoutait à la Loi sur l'assurance hospitalisation et les services diagnostiques de $1957^{2}$. Ce que l'on sait moins, c'est que la Commission plaçait l'assurance médicaments universelle au cœur de son régime. Le juge Emmet Hall avait même réfléchi à des façons spécifiques d'accommoder l'industrie pharmaceutique tout en mettant un frein à sa cupidité.

La Saskatchewan a géré avec succès un programme universel d'assurance médicaments complet pendant 12 ans à partir de 1975. Contrairement à d'autres régimes de soins de santé dont elle a été à l'avant-garde, le régime d'assurance médicaments de la Saskatchewan n'a pas suscité de financement en partenariat avec le gouvernement fédéral. C'est par ce type de financement en partenariat que le régime d'assurance hospitalisation de la Saskatchewan a essaimé vers le reste du Canada en 1957, suivi de l'assurance maladie en 1966. En effet, durant cette période, le gouvernement fédéral a commencé à se retirer de l'accord original de partage des coûts 50:50. Pour le programme d'assurance médicaments de la Saskatchewan, l'absence de financement fédéral est arrivée à un bien mauvais moment, car lors de sa mise sur pied, on imaginait mal que des patients puissent avoir besoin de médicaments tout au long de leur vie ou de plus d'un médicament à la fois. La plupart des Canadiens d'âge moyen aujourd'hui prennent au moins trois médicaments contre des maladies chroniques comme l'hypertension, l'hypercholestérolémie, le diabète, les brûlures d'estomac et l'arthrite. De nombreux patients prennent beaucoup plus que trois médicaments. Le programme universel d'assurance médicaments de la Saskatchewan a duré plus longtemps que prévu. De nos jours, les provinces offrent des programmes d'assurance médicaments sélectifs, par exemple, le programme d'assurance médicaments de l'Ontario pour les personnes âgées à faible revenu et l'extension du programme Assurance-santé Plus pour les citoyens ontariens de moins de 25 ans.

La fabrication des médicaments s'est automatisée. Nous disposons donc aujourd'hui de normes de production parmi les plus élevées au coût le plus faible de toute l'histoire de l'humanité. Tandis que les marges de profit 
sur les médicaments standards auraient dû diminuer grâce à cette technologie, l'industrie pharmaceutique continue d'être la plus profitable de toutes. Les compagnies pharmaceutiques prétendent y être parvenues grâce à l'innovation. Mais même si certains progrès ont été spectaculaires, de nombreux chercheurs croient que le principal moteur de l'innovation ressemble plus au désir de construire le nouveau modèle d'automobile de l'année ou de dessiner une nouvelle robe. Les médicaments plus anciens et moins coûteux ont été abandonnés pour des solutions de rechange plus récentes et plus chères, dont les soi-disant nouveaux atouts sont bien maigres pour justifier l'augmentation de leur coût pour le consommateur. Une production désordonnée a donné lieu à des pénuries critiques, désignées par l'euphémisme « rupture de stock », qui posent une menace pour la santé. À l'autre bout du spectre, de nouveaux traitements pour des maladies jusqu'alors incurables sont vendus à des prix astronomiques, souvent plus de 10 fois le salaire annuel du Canadien moyen. Même les assureurs privés ont du mal à garder la cadence, de sorte que de nombreux établissements remplacent les régimes qui remboursent le coût des médicaments par des comptes de dépenses santé limités.

Les régimes universels d'assurance médicaments provinciaux doivent pouvoir accéder aux médicaments. Une liste de médicaments nationale sécurisée pourrait bien être une responsabilité fédérale puisque c'est ce palier de gouvernement qui voit à l'homologation des médicaments. La création d'une liste de médicaments canadienne est à ce point cruciale pour notre sécurité qu'elle devrait être déclarée une question de sécurité nationale. Une telle déclaration permettrait au gouvernement fédéral d'utiliser les stratégies appropriées pour dompter l'appât du gain de l'industrie, sécuriser l'approvisionnement et demander au besoin la fabrication de médicaments avérés bénéfiques pour les maladies communes et les maladies rares. Une liste nationale de médicaments ne deviendrait pas un entrepôt massif, mais une stratégie d'approvisionnement de sources multiples, à la disposition des régimes d'assurance médicaments provinciaux, inspirée par plusieurs des principes formulés par le juge Hall.

En 1966, l'industrie de l'assurance soins de santé privée s'est sentie menacée au moment où les régimes d'assurance provinciaux ont pris la relève à une fraction du prix pour leur administration. Elle n'en a pas moins rebondi en offrant la couverture d'avantages supplémentaires. De même, il pourrait y avoir une place pour l'assurance privée afin qu'elle couvre certains a jouts, par exemple l'utilisation des produits originaux plutôt que des génériques ou certains traitements non couverts par l'assurance santé publique. Un formulaire national forcerait les experts à débattre de manière rationnelle du rapport coût-efficacité des médicaments plutôt que de laisser cette question et bien d'autres aspects de la disponibilité des médicaments aux caprices du marché. Le 20 juin 2018, le Canada a créé un Conseil consultatif sur la mise en œuvre d'un régime d'assurance médicaments national. Une liste de médicaments nationale offrirait au $\mathrm{D}^{\mathrm{r}}$ Eric Hoskins, président de ce Conseil, les moyens constitutionnels de réussir, comme avant lui, le juge Hall a ouvert la voie à l'assurance-santé il y a un demi-siècle.

\section{Vivian C. McAlister, M.B.}

Corédacteur en chef, fournal canadien de chirurgie

Intérêts concurrents : Aucun déclaré. Les opinions exprimées n'engagent que l'auteure et ne reflètent pas nécessairement celles de ses employeurs ou des établissements auxquels elle est affiliée.

DOI: $10.1503 /$ cjs.010518

\section{Références}

1. Harvey E. Le gouvernement Trudeau s'ingère dans les attributions des provinces. Can 7 Surg 2017;60:220.

2. Gouvernement du Canada. Le système de soins de santé du Canada. Disponible : www.canada.ca/fr/sante-canada/services/systeme-soins -sante/rapports-publications/regime-soins-sante/canada.html (consulté le 23 juin 2018).

3. Justice Emmett Hall. Farm Forum (entrevue), 2 nov. 1964. CBC Digital Archives. Disponible: www.cbc.ca/player/play/1801207811 (consulté le 23 juin 2018). 